\title{
Periodic Structures Fabricated By STED-DLW Stereolithography: Morphology and Optical Properties
}

\section{D.A. Kolymagin ${ }^{1}$, R.D. Zvagelsky¹, D.A. Chubich¹, and A.G. Vitukhnovsky ${ }^{1,2,3}$}

${ }^{1}$ Moscow Institute of Physics and Technology MIPT, Dolgoprudny

${ }^{2}$ Lebedev Physical Institute of the RAS, Moscow

${ }^{3}$ National Research Nuclear University MEPhl (Moscow Engineering Physics Institute), Kashirskoe shosse 31, Moscow, 115409, Russia

\section{Abstract}

Two-dimensional (photonic antennas) and three-dimensional (photonic crystals) periodic polymer structures were fabricated by STED-DLW stereolithography. Their morphological features were studied using scanning electron microscopy and atomic force microscopy; also their optical properties were investigated by Fourier

Corresponding Author:

D.A. Chubich

chubich.conference@gmail.com

Received: 28 January 2018

Accepted: 15 March 2018

Published: 25 April 2018

Publishing services provided by

Knowledge E

(c) D.A. Kolymagin et al. This article is distributed under the

terms of the Creative Commons

Attribution License, which permits unrestricted use and redistribution provided that the original author and source are credited.

Selection and Peer-review under the responsibility of the PhIO Conference Committee. spectroscopy and confocal microscopy.

Keywords: STED-DLW stereolithography, optical properties, luminescent mapping, 3 D structures

\section{Introduction}

The development of efficient and low-cost methods for fabrication of 3D and 2.5D nanostructures appear to be vitally important. Recently the Direct Laser Writing (DLW) method has gained a certain momentum. This technique is based on two-photon polymerization that occurs when a photoresist is irradiated by a femtosecond laser.

It is possible to increase the resolution of the DLW at constant excitation wavelength using the idea of STED (STimulated Emission Depletion) to reduce the size of the effective excited area. The principle of the method resides in utilization of a second depletion laser to stimulate radiative transitions on the edges of the focal spot. Consequently, the size of the excited area is reduced due to the effect of the depletion electromagnetic field.

In 2014 the Nobel Prize in chemistry was awarded for implementation of the stimulated depletion technique in microscopy. This method provides the way to overcome 
the constraints imposed by diffraction (Abbe limit) and achieve superior spatial resolution (as high as $50 \mathrm{~nm}$ ) [1]. On the other hand, implementation of the STED technique in lithography could significantly extend the technological potential for fabrication of 3D structures using additive technologies (STED-DLW stereolithography). This idea was proposed by the authors in 2012 [2].

Currently the market of printed electronics develops rapidly and according to expert estimates its total turnover will be as large as 100 billions of dollars by 2020 . The developments in this field cover both single-component (polymers and metals) and multicomponent printing. The latter, in turn, involves compositions of different materials creating new opportunities for development of conceptually new advanced devices. Three-dimensional structures fabricated with the use of an additive technology (see, eg, [3]) as well as the nanoobjects obtained by colloidal synthesis [4-7] and epitaxial growth [8-10] which are fairly widespread nowadays appear to be quite promising for microelectronic and nanophotonic applications [11-14].

\section{Methods}

The employment of femtosecond two-photon polymerization with introduction of STED laser radiation provides a means to fabricate the constructions with predefined shapes using computer-controlled precision 3D-nanopositioners [15, 16]. A crucial role in lithography is played by a photoinitiator which is essentially a dye that is able to promote photopolymerization and/or photoreduction (in the case of metals) reactions. However, not every dye is suitable for the STED technique. The appropriate photoinitiator should reveal a number of optical and photochemical properties.

Diffraction imposes limitations on pattering and recognition of spatial structures with sizes significantly smaller than the wavelength of the light they are exposed to (Abbe diffraction limit: $\left.\Delta \mathrm{x}_{A b b e}=\lambda /(2 n \sin \alpha)\right)$. Metamaterials and near-field optical microscopy are conventionally employed to break the diffraction limit [17].

In recent years several approaches providing an opportunity to break the diffraction limit in far-field fluorescence microscopy have appeared [1]. With their improved resolution these ideas advance us beyond the results achieved using the existing techniques [18].

Let us assume that a light-absorbing medium can make transitions between two states $A$ and $B$ (see Fig. 1). In the simplest case $A$ is the ground state and $B$ is the excited state. In our situation the transition $A \rightarrow B$ is to be an allowed one with regard to the selection rules. 
With the rate constants for the transitions given as $k_{A B}$ and $k_{B A}$ we obtain the following relationship describing the populations $N_{A}$ and $N_{B}$ of the levels:

$$
d N_{A} / d t=-k_{A B} N_{A}+k_{B A} N_{B}=-d N_{B} / d t
$$

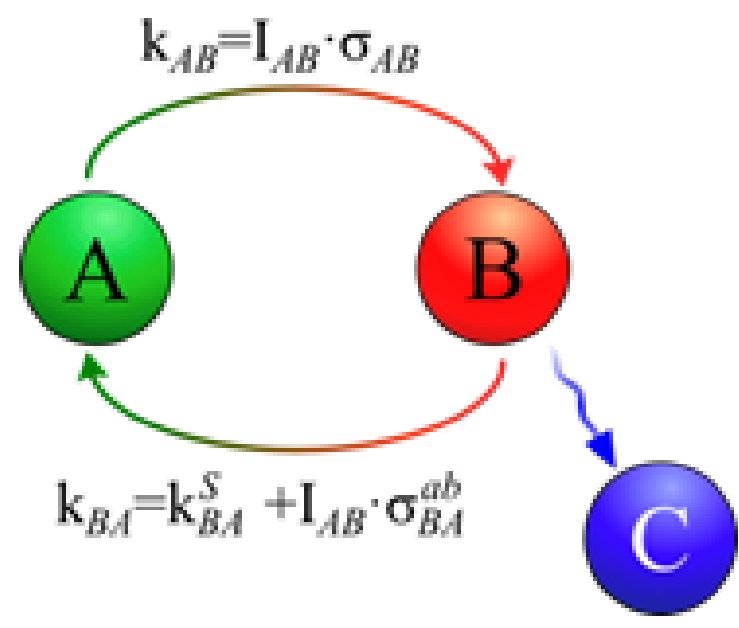

Figure 1: Cycle of states for photoinitiator. $k_{A B}$ is rate constant for $A \rightarrow B$ transition; $l_{A B}$ is intensity of incident radiation, $\sigma_{A B}$ is transition cross section; $k_{B A}$ is rate constant for $B \rightarrow A$ transition including contributions from spontaneous component $k_{A B} S$ and component induced by incident radiation $I_{A B} ; \sigma_{B A}{ }^{a b}$ is transition cross section.

If the medium was initially in the state $A$ then the probability to observe it in the state $A$ after time $t$ is

$$
N_{A}(t)=k_{A B}\left(k_{B A} / k_{A B}+\exp \left[-\left(k_{A B}+k_{B A}\right) t\right] /\left(k_{A B}+k_{B A}\right)\right)
$$

For $\mathrm{t}>5 \cdot\left(k_{A B}+k_{B A}\right)^{-1}$ we neglect the exponential term and take $N_{A}{ }^{\infty}=k_{B A} /\left(k_{A B}\right.$ $\left.+k_{B A}\right)$. Let us consider the transition to the state $B$. The transition $A \rightarrow B$ occurs under exposure to light with the wavelength of $\lambda_{A B}$ and the corresponding rate constant is $\sigma_{A B} l_{A B}$, where $\sigma_{A B}$ is the transition cross section and $I_{A B}$ is the local number of photons per area. In the most general case suppose that $I_{A B}$ induces the transition $B \rightarrow A$ with the cross section $\sigma_{B A}{ }^{a b}<\sigma_{A B}$. Therefore, the transition $B \rightarrow A$ includes a contribution from the spontaneous component $k_{B A}{ }^{S}$ and an induced one and, consequently, $k_{A B}=$ $k_{A B}{ }^{S}+\sigma_{B A}^{a b} I_{A B}$. This implies that

$$
N_{A}{ }^{\infty}=\left(\sigma_{B A}{ }^{a b} I_{A B}+k_{B A}{ }^{S}\right) /\left(\left(\sigma_{A B}+\sigma_{B A}{ }^{a b)}\right) I_{A B}+k_{B A}{ }^{S}\right)
$$

For intensities $I_{A}>>k_{B A}{ }^{S} /\left(\sigma_{A B}-\sigma_{B A}{ }^{a b}\right) \equiv I_{A B}{ }^{\text {sat }}$ we obtain $\bar{N}_{A}^{\infty}=\sigma_{B A}^{a b} /\left(\sigma^{A B}+\sigma_{B A}^{a b}\right)$.

Let the transition $A \rightarrow B$ be induced by the light field with the spatial intensity distribution that contains a local zero point: $I_{A B}(\vec{r})=I_{A B}^{\max }(\vec{r}), \vec{r}_{0}=0 ; f(\vec{r}=x, y, z)$ is a normalized function describing the diffraction-limited intensity distribution. If we take $I_{A B}{ }^{\max }>>I_{A B}$ sat we obtain $N_{A}=\bar{N}_{A}$ at each point of space excluding the near 
neighborhood of $\vec{r}_{0}$ point, where $N_{A}{ }^{\infty}=1$. Thus, we can create an arbitrarily narrow region of the medium in the state $A$ incorporated into the area with a significant fraction of the molecules in the state $B$. It is important that the size of the $A$-region does not depend either on $\lambda_{A B}$ or on the peculiarities of $f(\vec{r})$. It makes no difference whether $f(\vec{r})$ describes a Laguerre-Gaussian mode, or a standing wave, or any other function if there is at least one local zero point involved.

In ordinary cases $I_{A B}{ }^{\text {max }}$ and $I_{A B}$ sat are finite and $f(\vec{r})$ is a certain function. In the simplest case regarding a standing wave $f(x)=\sin ^{2}\left(2 \pi x / \lambda_{A B}\right)$ full width at half-maximum for a narrow $A$-region can be obtained as follows: $I_{A B}{ }^{\max } f(x)=I_{A B}$ sat

$$
\Delta x \geq \frac{\lambda_{B A}}{\pi} \arcsin \left(\sqrt{\frac{k_{A B}^{S}}{\left(\sigma_{B A}-\sigma_{A B}^{b a}\right) I_{A B}^{\mathrm{max}}}}\right)=\frac{\lambda_{B A}}{\pi} \arcsin \left(\varsigma^{-1 / 2}\right) \approx \frac{\lambda_{B A}}{\pi \sqrt{\varsigma}}
$$

Possible values for the saturation factor are $\varsigma=I_{A B}{ }^{\max } / I_{A B}$ sat $>>1$. If $\varsigma=10000$ we have $\Delta \mathrm{x} \approx \lambda_{A B} / 100$. However, theoretically it is even possible to reduce the spot size down to molecular scale.

In our case the method under consideration is applied in stereolithography. If the utilized medium can make a transition from the state $A$ to a stable state $C$ a new concept for laser writing (nanolithography) can be deduced with no regard to diffraction limit.

First, using the intensity profile $I_{A B}(\vec{r})=I_{A B}^{\max } f(x)$ we promote the transition of the medium from the state $A$ to the state $B$ which does not induce polymerization. And the region $\Delta \bar{x} \ll \lambda_{A B}$ of the medium stays in the state $A$. Then we induce the transition of the molecules that are still in the state $A$ to the stable state $B$. While the role of the saturable transition $A \rightarrow B$ resides in specification of the position and width of the structures, the role of the state $B$ itself is to prevent the material from polymerization. Therefore, the main feature of writing is the presence of an intermediate preserving state $B$ which is not prone to polymerization.

It is important that the width $\Delta \bar{x}$ and the distance between the elements can be scaled at will. As a result, such approach provides a means to obtain the structures of any size regardless of the excitation wavelength.

Within this new concept diffraction is not the fundamental limiting factor for achieved resolution; in fact, the resolution is solely limited by the saturation level of the medium. Therefore, the task is to find a saturable transition with low saturation intensity $I_{A B}$ sat.

With employment of appropriate materials this idea can be extended to diffractionunlimited lithography. In this case the resolution depends only on the saturation level and is described by a simple formula. While the effectiveness of this method was 
demonstrated in microscopy by stimulated emission depletion [1], the generalization for the case of STED-DLW stereolithography should take into account the additional physical requirements for the photoinitiator associated with the effective start of the photostimulated chemical reaction only within the region in which the dye molecules remains in an excited state after depletion.

Moreover, using the lift-off technique it is possible to achieve subsequent metallization of the template fabricated by stereolithography with formation of a silver/gold structure. Such structures are of special interest primarily due to plasmonic effects.

STED-DLW stereolithography also can be used to create hybrid structures containing Ag nanoparticles. Utilizing the depletion laser allows one to reduce the feature size from $570 \mathrm{~nm}$ (DLW technology) to $220 \mathrm{~nm}$ (STED-DLW technology) [see Fig.2].

A system for the STED-DLW stereolithography was built on the basis of a femtosecond Ti:Sapphire laser with pulse duration of $50 \mathrm{fs}$ and emission wavelength tunable over 720-860 nm range. The system involved a continuous-wave depletion laser emitting at $532 \mathrm{~nm}$ as well. Optimization of the photoresist composition and parameters of the STED-DLW stereolithography is described elsewhere [16].

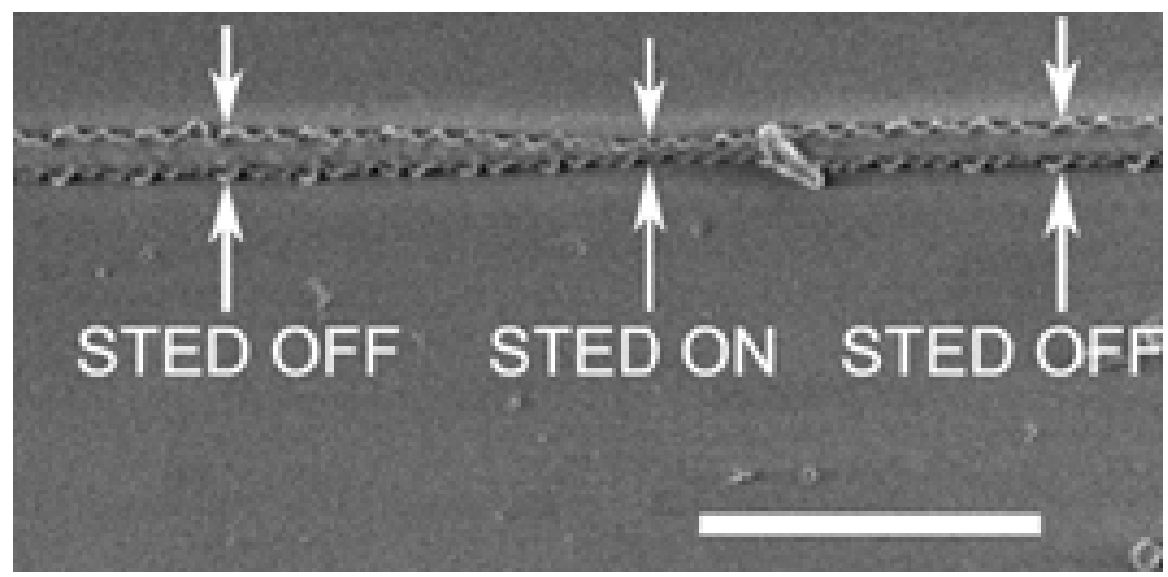

Figure 2: SEM image of hybrid structure, created with STED-DLW stereolithography (STED ON) and DLW (STED OFF). Scale bar $5 \mu \mathrm{m}$.

\section{Results and discussion}

Using the improved technique for direct laser writing we fabricated 2.5D (see Fig. 3) and $3 \mathrm{D}$ periodic structures with various spatial periods.

The morphology of the obtained structures was studied with the use of scanning electron microscopy (SEM) and atomic force microscopy (AFM). Alongside with that, 


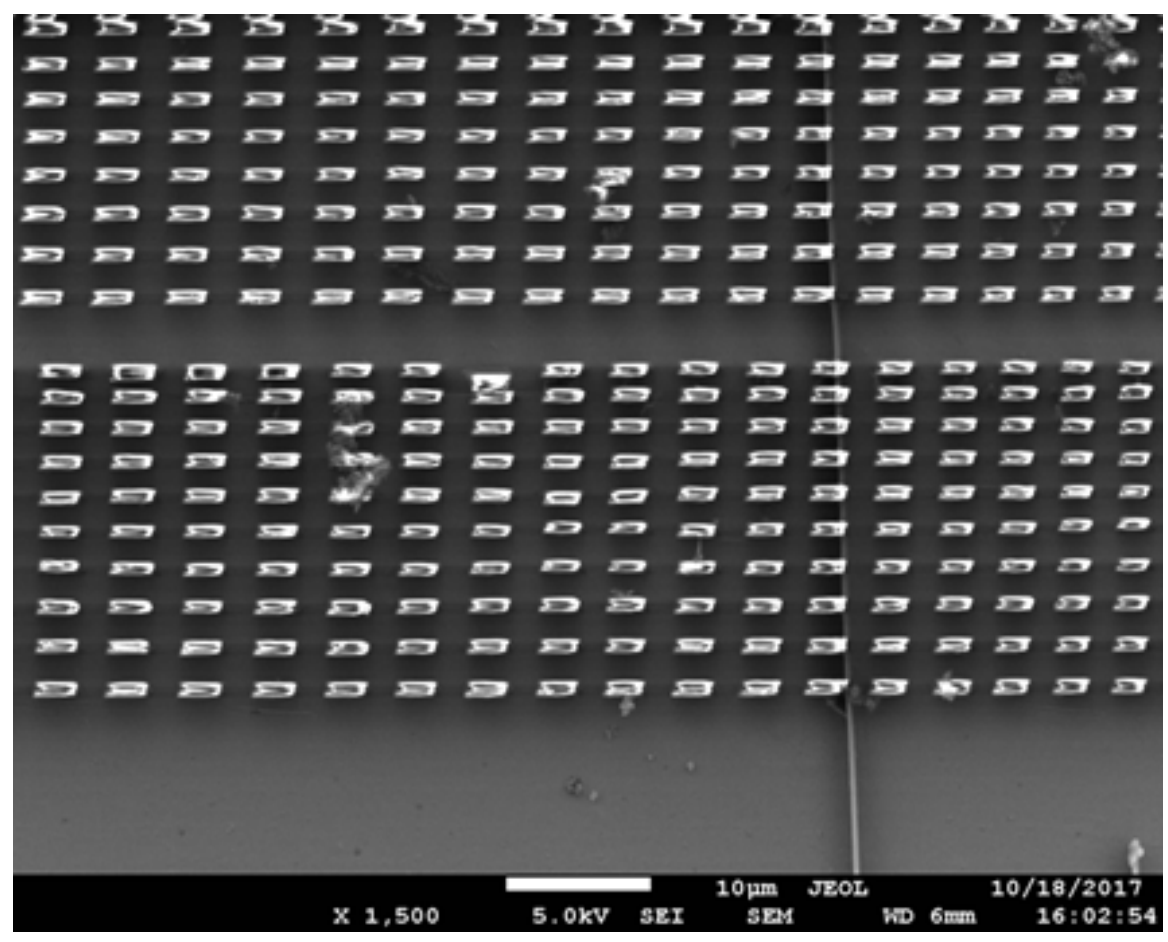

Figure 3: SEM image of periodic step-like structures.

optical properties of the structures were investigated by Fourier-spectrometry providing a means to monitor and control the polymerization degree using the intensity of the IR-absorption line associated with the $C=C$ stretching modes [19]. Confocal laser scanning microscopy was employed as well.

Using the STED-nanolithography system we fabricated periodic step-like structures with various periods. The SEM image of the obtained structures is presented in Fig. 3.

Photopolymerization results in the presence of the residual photoinitiator (DETC dye, 7-diethylamino-3-thenoyl-coumarin) within the obtained nanostructures. The concentration of DETC is sufficient to detect its photoluminescence using a confocal microscope and time-correlated single photon counting technique.

The results of luminescence mapping of the structures excited via one-photon absorption at the wavelength of $375 \mathrm{~nm}$ are presented in Fig. 4. The presence of DETC extends the application potential of our system for utilizing it in not only STED-DLW stereolithography but also in STED-microscopy. This fact facilitates the development of an integrated unique complex providing a means for both fabrication and investigation of the structures with spatial resolution as high as $30 \mathrm{~nm}$.

Spectral-kinetic properties of the dye within the periodic structures were investigated. The histogram of average lifetimes for the excited state of the DETC dye in the studied structures is presented in Fig. 5 . 

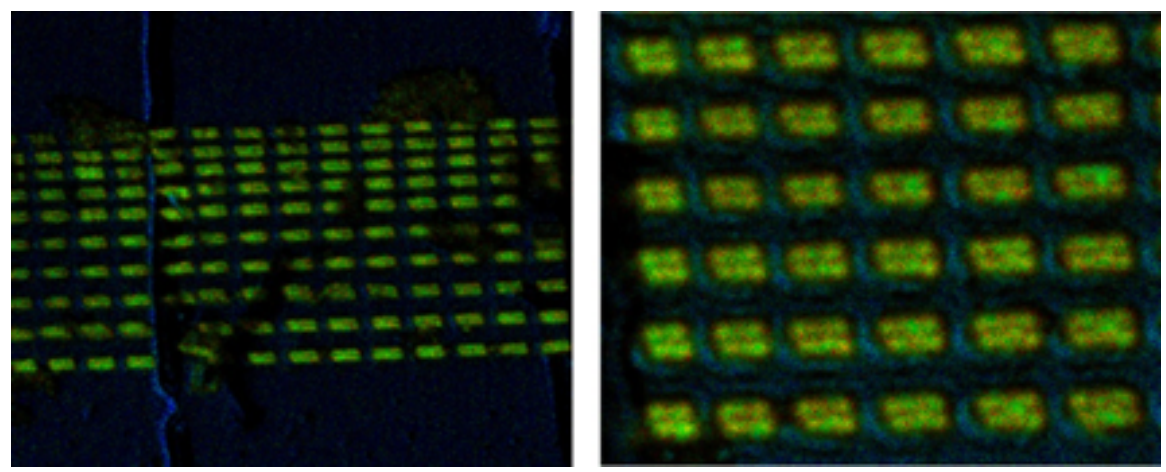

Figure 4: luminescent image of periodic structure containing DETC. Right: scaled-up image of fragment of periodic luminescent structure. Lateral periods of dashes are $d x=3.5 \mu \mathrm{m}$ and $d y=3 \mu \mathrm{m}$.

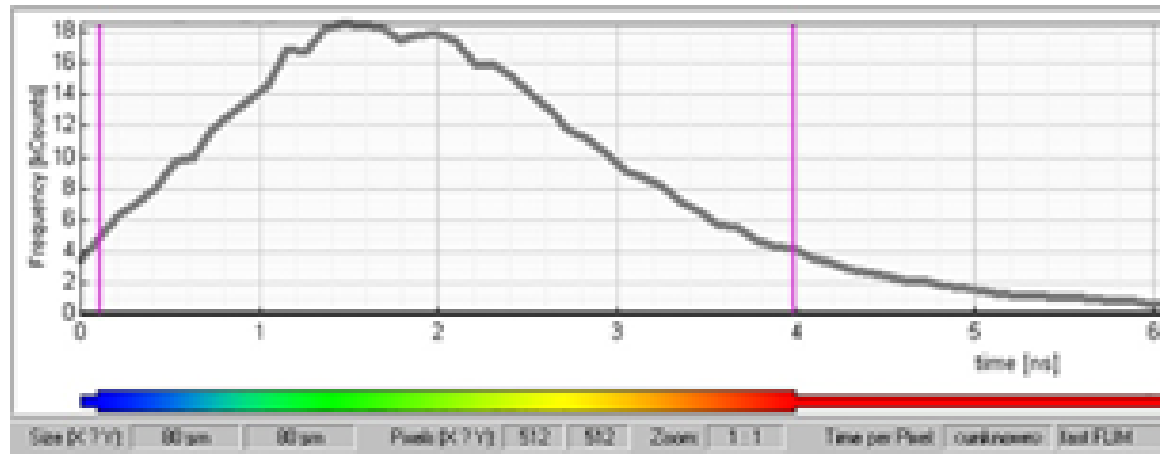

Figure 5: Histogram of average lifetimes for excited state of DETC in fabricated structures.

On the basis of the analysis of luminescence decays for the same structures we obtain the average lifetimes of $2.3 \pm 0.3 \mathrm{~ns}$ for the excited state.

\section{Conclusion}

A STED-DLW stereolithographer was developed and its potential for fabrication of 2.5D and $3 \mathrm{D}$ structures was demonstrated. Submicron spatial resolution was achieved by employing the laser polymerization method with local suppression of the photostimulated reaction by the STED laser beam. Utilization of the STED-DLW stereolithographer will facilitate the development of additive technologies for fabrication of $2 \mathrm{D}, 2.5 \mathrm{D}$ and $3 \mathrm{D}$ objects with nanometer resolution. Such structures will be suitable for a wide range of novel applications including microoptics and photonics, microfluidics, scaffold fabrication (tissue engineering), maskless lithography, fast prototyping for electronics industry and more.

State-of-the-art technology of Surface-Enhanced Infrared Absorption (SEIRA) provides a means to increase the sensitivity of absorption by five orders of magnitude as compared to conventional methods of transmission IR spectroscopy. The effect is 
achieved by using a metallic periodic substrate [20]. It is possible to create the mask required for fabrication of such photonic antennas using the methods described above.

The results of luminescence mapping indicate that after photopolymerization the obtained structures still contain the amount of the DETC dye which is sufficient for subsequent employment of the STED-microscopy technique providing a means to develop an integrated lithography-measurement method with the spatial resolution as high as $30 \mathrm{~nm}$ which cannot be achieved with the use of other lithographic techniques unless high-vacuum technology is involved.

\section{Acknowledgments}

The authors are grateful to S.P. Eliseev, PhD, for his help with the luminescence measurements and to V.V. Sychev for valuable discussions and technical assistance.

This work was performed using the equipment of the MIPT Shared Facilities Center and with financial support from the Ministry of Education and Science of the Russian Federation (Grant No. RFMEFI59417Xo014). The development of the technology for fabrication of $3 \mathrm{D}$ structures was funded by the Russian Science Foundation under project no. 15-19-00205 and the financial support for the research work was granted by the Russian Science Foundation under project no. 17-79-20418.

\section{References}

[1] Hell S.W., Wichmann J. Opt. Lett., 19, 780 (1994).

[2] Glubokov D.A., Sychev V.V., Vitukhnovskii A.G., Taidakov I.V. "Method of manufacturing resistive masks for nanolithography," RF Patent No. 2510632 (2012).

[3] Wong K.V., Hernandez A. ISRN Mech. Eng., 2012, 1 (2012).

[4] Peng X., Manna L., Yang W., Wickham J., Scher E., Kadavanich A., Alivisatos A.P. Nature, 404, 59 (2000).

[5] Vitukhnovsky A.G., Shul'ga A.S., Ambrozevich S.A., Khokhlov E.M., Vasiliev R.B., Dirin D.N., Yudson V.I. Phys. Lett. A, 373, 2287 (2009).

[6] Katsaba A.V., Fedyanin V.V., Ambrozevich S.A., Vitukhnovsky A.G., Lobanov A.N., Selyukov A.S., Samatov I.G., Brunkov P.N. Semiconductors, 47, 1328 (2013)

[7] Vasiliev R.B., Sokolikova M.S., Vitukhnovskii A.G., Ambrozevich S.A., Selyukov A.S., Lebedev V.S. Quantum Electron., 45, 853 (2015). 
[8] Sugiyama T., Kuwahara Y., Isobe Y., Fujii T., Nonaka K., Iwaya M., Takeuchi T., Kamiyama S., Akasaki I., Amano, H. Appl. Phys. Express, 4, 015701 (2010).

[9] Chung R.B., Wu F., Shivaraman R., Keller S., DenBaars S.P., Speck J.S., Nakamura S. J. Cryst. Growth, 324, 163 (2011).

[10] Amano H. Jpn. J. Appl. Phys., 52, 050001 (2013).

[11] DenBaars S.P., Feezell D., Kelchner K., Pimputkar S., Pan C.C., Yen C.C., Tanaka S., Zhao Y., Pfaff N., Farrell R., Iza M., Keller S., Mishra U., Speck J.S., Nakamura S. Acto Mater., 61, 945 (2013).

[12] Selyukov A.S., Vitukhnovskii A.G., Lebedev, V.S., Vashchenko A.A., Vasiliev R.B., Sokolikova M.S., J. Exp. Theor. Phys., 120, 595 (2015).

[13] Kurokawa H., Kaga M., Goda T., Iwaya M., Takeuchi T., Kamiyama S., Akasaki I., Amano H. Appl. Phys. Express, 7, 034104 (2014).

[14] Selyukov A.S., Isaev A.A., Vitukhnovsky A.G., Litvak V.L., Katsaba A.V., Korshunov V.M., Vasiliev R.B. Semiconductors, 50, 947 (2016).

[15] Eliseev S.P., Korolkov A.E., Vitukhnovsky A.G., Chubich D.A., Sychev V.V. Nanotechnol. Russ., 11, 200 (2016).

[16] Vitukhnovsky A.G., Chubich D.A., Eliseev S.P., Sychev V.V., Kolymagin D.A., Selyukov A.S. J. Russ. Laser Res., 38, 375 (2017).

[17] Huang B., Bates M., Zhuang X. Annu. Rev. Biochem., 78, 993 (2009).

[18] Bechhoefer J. Am. J. Phys., 83, 22 (2015).

[19] Chubich D.A., Kolymagin D.A., Kazakov I.A., Vitukhnovsky A.G. Bull. Russ. Acad. Sci.: Phys., in press (2018).

[20] Jahn M., Patze S., Hidi I.J., Knipper R., Radu A.I., Mühlig A., Yüksel S., Peksa V., Weber K., Mayerhöfer T., Cialla-May D., Popp J. Analyst, 141, 756 (2016). 